\title{
Severe amitriptyline poisoning treated successfully with combined hemoperfusion and hemodialysis
}

\author{
Ding-Fan Wu ${ }^{1}$ \\ (iD) Tian-You Zhou ${ }^{1}$ \\ (iD) jiang-Shan Zhang ${ }^{2}$
}

1. Department of Emergency, First Affiliated Hospital of Guizhou University of Traditional Chinese Medicine, Guiyang 550001 , Guizhou, China 2. Intensive Care Unit, First Affiliated Hospital of Guizhou University of Traditional Chinese Medicine, Guiyang 550001, Guizhou, China

Dear Editor,

We read with great interest the review by Camargo et al. ${ }^{1}$ and the study by Günalay et al. $^{2}$ in which they summarize that the clinical presentations of uremic neuropathy are broad and non-specific and that malnutrition is associated with quality of life in hemodialysis and peritoneal dialysis patients. Their opinion was helpful for clinical work.

In our clinical study, a 15-year-old male patient that progressed to coma and limb convulsion due to a one-time oral administration of 100 amitriptyline tablets (25mg/ tablet) was enrolled in our hospital. The patient's family found and sent him to the local county hospital for treatment 6 hours after the onset. Since 6 hours had already gone by, the county hospital did not perform gastric lavage, but direct hemoperfusion treatment, rehydration, anti-epilepsy, awakening, and other treatments. After treatment for 2 days, the conscious state and convulsive state of the patient had not improved, so he was transferred to the emergency ICU of our hospital. The patient was GCS6, with bilateral pupil diameter of about $4.0 \mathrm{~mm}$, slow light reflexes, paroxysmal, limb tics last about 3 minutes each time, fever of $38.5^{\circ} \mathrm{C}$, electrocardiogram, sinus tachycardia, BP 120/80 mmHg, blood gas analysis: PH 7.40, PCO2 $34.20 \mathrm{mmHg}$ and PO2 $103.00 \mathrm{mmHg}$, cLac1.30 / L, ABE - 2.90, HCO3 - 20.70 / L, liver and kidney function of electrolyte in the normal range. After admission, continuous gastrointestinal decompression and about $300 \mathrm{ml}$ of pale dark green gastric material were immediately obtained, and filtrate from the German multifil rate and HP330 perfusion apparatus were used to maintain CRRT after two hours of perfusion and CVVH mode. After 6 hours, hemoperfusion performed again, and phenobarbital antiepileptic therapy administered at the same time. After 12 hours of treatment, the pupil diameter of the patient was restored to $3.0 \mathrm{~mm}$, 
and the patient became sensitive to light relaxation. After 24 hours, the consciousness of the patient was restored, and he was able to ask and answer questions and follow instructions to shake hands; limb convulsions did not occur again. The patient was conscious 48 hours later and with a GCS14 score.The patient was transferred out of the EICU after 4 days of treatment and discharged 7 days later. When discharged from the hospital, he had clear consciousness, could answer questions accurately, had a GCS515 score, and stable vital signs.

In conclusion, various blood purification models have been reported to be effective treatments ${ }^{3}$.
Although there is a lack of large sample data analysis to support it, blood purification therapy, especially hemoperfusion therapy, should be used as early as possible.

\section{REFERENCES}

1. Camargo CRS, Schoueri JHM, Alves BDCA, Veiga GRLD, Fonseca FLA, Bacci MR. Uremic neuropathy: an overview of the current literature. Rev Assoc Med Bras (1992). 2019;65(3):469-74.

2. Günalay S, Öztürk YK, Akar H, Mergen H. The relationship between malnutrition and quality of life in haemodialysis and peritoneal dialysis patients. Rev Assoc Med Bras (1992). 2018;64(9):845-52.

3. Sheng C, Zhang Z, Jia Y, Li Y. Changes in serum cardiac myosin light chain 1 levels in children with fulminant myocarditis during continuous blood purification. Rev Assoc Med Bras (1992). 2017;63(10):904-9. 